\title{
Cambios Dimensionales en Técnicas de Preservación Alveolar Bartee y Bio-Col con Xenoinjerto Inteross ${ }^{\circledR}$
}

\author{
Dimensional Changes in Bartee and Bio-col Ridge \\ Preservation Technique Using Xenograft InterOss ${ }^{\circledR}$
}

\author{
Melanie Karoly Elizalde Mota'; Cindy Hernández Romero²; \\ Valeria Magali Rocha Rocha ${ }^{3}$ \& Veronica Anuette Mayoral García ${ }^{4}$
}

ELIZALDE, M. M. K.; HERNÁNDEZ, R. C.; ROCHA, R. V. M.; MAYORAL, G. V. A. Cambios dimensionales en técnicas de preservación alveolar bartee y bio-col con xenoinjerto inteross ${ }^{\circledR}$. Int. J. Odontostomat., 15(2):370-376,2021.

RESUMEN: La preservación de las dimensiones y contorno del reborde alveolar posterior a una extracción es de suma importancia para evitar problemas subsecuentes para la colocación de un implante dental. El objetivo de este estudio fue comparar los cambios dimensionales mediante el análisis en tomografía computarizada de haz cónico (CBCT) en sitios pre y post preservados con técnica Bartee y Bio-Col con xenoinjerto a los 6 meses de cicatrización. En un paciente de 62 años, se realizaron 6 preservaciones alveolares en órganos dentarios uniradiculares, con diagnóstico periodontal sin esperanza, grupo A la técnica de preservación alveolar Bartee $(n=3)$ y grupo B la técnica de preservación alveolar Bio-Col $(n=3)$. Se utilizó xenoinjerto (InterOss $®$ Anorganic Cancellous Bone Graft Granules 0,25- 1mm Sigma graft) en ambos grupos. En el grupo A se colocó injerto óseo en la totalidad del alveolo asistido por una membrana no reabsorbible de politetrafluoroetileno denso (Cytoplast TM Regentex TXT-200 singles, Osteogenics Biomedical Inc, Lubbock, Texas). En el grupo B se colocó el injerto óseo en $3 / 4$ del alveolo y el último $V / 4$ del alveolo fue ocupado por apósito de colágeno reabsorbible (CollaPlug ${ }^{\circledR}$ Zimmer biomet). Se registraron mediciones obtenidas mediante CBCT inicial previa a la realización de preservaciones alveolares y se tomó una segunda CBCT a los 6 meses de cicatrización obteniendo la medición en sentido vertical y horizontal, coincidiendo en el plano de corte. Se observó mediante el análisis dimensional en grupo A y Grupo B obteniendo en sentido vertical un $13,58 \%$ y $20,76 \%$ de reabsorción y en sentido horizontal $13,45 \%$ y $15,72 \%$ respectivamente a los 6 meses de cicatrización, utilizando xenoinjerto por lo que no existe diferencia estadísticamente significativa en cuanto a los cambios dimensionales entre ambas técnicas $p>0,05$. La preservación alveolar proporciona una estabilidad dimensional contrarrestando el proceso de reabsorción fisiológica, siendo una opción predecible.

PALABRAS CLAVE: pérdida ósea alveolar, remodelación ósea, xenoinjerto.

\section{INTRODUCCIÓN}

Posterior a la extracción de un diente sucede un proceso fisiológico de remodelación que involucra tanto a los tejidos duros como blandos, lo cual resulta en una contracción dimensional de la cresta alveolar tanto en altura como en anchura. Existen múltiples variables a considerar, incluidas entre ellas; las dimensiones del alveolo, el grosor de la mucosa, factores metabólicos, carga funcional, entre otros (Maiorana et al., 2017).
La preservación de las dimensiones y contorno del reborde alveolar posterior a una extracción es de suma importancia para evitar problemas subsecuentes que dificulten la colocación de un implante dental y su posterior rehabilitación (Shakibaie-M, 2013).

Las fuerzas funcionales como el bruxismo, el desgaste completo de las dentaduras protésicas y las fuerzas de mordida mayores, han sido relacionadas como

\footnotetext{
${ }^{1}$ Residente del Posgrado de Periodoncia Universidad Popular Autónoma del Estado de Puebla, México.

2 Directora Académica Posgrado Periodoncia Universidad Popular Autónoma del Estado de Puebla, México.

${ }^{3}$ Docente e Investigadora. Centro Interdisciplinario de Posgrados, Universidad Popular Autónoma del Estado de Puebla, México.

${ }^{4}$ Docente Posgrado Periodoncia Universidad Popular Autónoma del Estado de Puebla, México.
} 
factores contribuyentes en la aceleración de pérdida ósea. Dichas fuerzas, según la ley de Wolff, causan un patrón distintivo de formación y mantenimiento óseo a lo largo de las líneas de estrés. Los cambios estructurales en el hueso ocurren a través de procesos celulares de reabsorción osteoclástica y deposición osteoblástica de colágeno y la posterior mineralización de la matriz de colágeno (Bartee et al., 2001a,b).

Una revisión sistemática, la cual analizó los cambios dimensionales de la cresta alveolar, reportó una pérdida ósea horizontal que oscila entre el $29 \%$ y el $63 \%$ y una pérdida ósea vertical entre el $11 \%$ y el $22 \%$ a los 6 meses posterior a la extracción dental (Tan et al., 2012).

El término "Preservación del reborde alveolar" (ARP, por sus siglas en inglés), ha sido definido como "cualquier procedimiento realizado en el momento o después de una extracción, diseñada para minimizar la reabsorción externa de la cresta y maximizar la formación ósea dentro del alvéolo" (Hämmerle et al., 2012).

Se ha demostrado que la preservación de la cresta alveolar no previene completamente la pérdida ósea, únicamente ayuda a reducir la magnitud de dicha pérdida dependiendo de diversos factores tales como los biomateriales, la técnica utilizada, entre otros (Jamjoom \& Cohen, 2015).

Existen diversas técnicas de preservación alveolar, siendo las más populares la técnica de Bartee y la técnica Bio-Col. La técnica de Bartee es asistida por una membrana no reabsorbible politetrafluoroetileno densa (PTFE-d), diseñada específicamente para su uso en procedimientos regenerativos, que no requieren de un cierre primario, evita la migración de partículas del injerto óseo al mismo tiempo que previene la migración de tejidos blandos y epiteliales durante el proceso de cicatrización, siendo un requisito indispensable en procedimientos de regeneración ósea guiada, el bloqueo de la migración epitelial en los defectos óseos da como resultado una regeneración de hueso potencializada; mediante la repoblación celular selectiva de la herida por células osteoprogenitoras, gracias a la concentración local de factores de crecimiento biológicos. La cinética de cicatrización en los sitios de extracción puede verse de manera similar, ya que existe una tendencia a la invaginación de los tejidos blandos y a la formación de tejido fibroso en el tercio coronal del alveolo que no requiere el cierre primario por lo que se asume que facilita la conservación de la encía queratinizada y la arquitectura gingival, así como también una mejora en el color de la cicatrización proporcionado por un tejido óseo altamente vascularizado libre de fibrosis o inflamación crónica a los 6 meses de cicatrización en preservación alveolar (Fotek et al., 2009).

En cambio la técnica Bio-Col, se utiliza un apósito de colágeno reabsorbible, el cual puede actuar como una membrana reabsorbible, promueve la regeneración ósea guiada durante al menos 30 días, se ha utilizado como un biomaterial para procedimientos de preservación alveolar, proporciona resultados alentadores en el mantenimiento de los tejidos blandos y minimiza la resorción en el alveolo, es tan efectivo como otras membranas de regeneración tisular guiada en la inhibición de la migración epitelial y en la promoción de una nueva unión del tejido conectivo (Position Paper: Periodontal Regeneration, 2005). Así mismo, el uso de apósito de colágeno reabsorbible es preferible debido a su proceso de absorción fisiológica y alta biocompatibilidad con los tejidos orales (Sclar et al., 1999), por otra parte, el colágeno es un agente hemostático y posee la capacidad de estimular la agregación de plaquetas y mejorar el enlace de fibrina, lo que puede conducir a la formación inicial de coágulos, la estabilidad y la maduración. Se ha demostrado que el colágeno es quimiotáctico para fibroblastos en estudios in vitro; dicha propiedad en términos generales podría mejorar la migración celular y poder así promover el cierre primario de la herida la cual se considera fundamental para lograr el crecimiento óseo (Wang et al., 2004).

Se han reportado resultados positivos con el uso membrana de colágeno reabsorbible en preservación alveolar, presenta cambios morfológicos mínimos a los 6 meses posterior a la extracción (Wang et al.) lo que se relaciona no solo una morfología adecuada sino que también con una densidad ósea que permite la preparación de la osteotomía en implantes dentales; así como también se ha logrado preservar el perfil de tejidos blandos presentes en el órgano dentario previo a la extracción dental (Fowler \& Whicker, 2004).

El mineral óseo bovino desproteinizado (DBBM) es un xenoinjerto utilizado como sustituto óseo obtenido de un donante de otra especie, el cual ha sido privado de todos sus componentes orgánicos a través de procesos de alta temperatura con el fin de minimizar la respuesta inmune, el cual mantiene una estructura cristalina, porosa y un contenido de carbonato similares a los minerales óseos humanos y debido a la similitud con la estructura ósea nativa, se afirma que 
proporciona un entorno osteoconductivo favorable durante el proceso de regeneración ósea (Bartee et al.). Si bien es cierto que el xenoinjerto experimenta un proceso de reabsorción mucho más lento, no se sabe si esto interfiere con la formación vital del hueso. Se habla de un porcentaje de xenoinjerto residual que oscila entre un 14,8 a un 36,6 \% (Chan et al., 2013). La capacidad de prevenir cambios dimensionales en los alveolos injertados con xenoinjerto depende de las propiedades de las partículas del injerto óseo que aún se pueden observar en el sitio edéntulo, incluso 7 meses posterior de haber realizado la preservación alveolar. Sin embargo, las muestras injertadas postextracción con xenoinjerto mostraron una calidad y cantidad de hueso clínicamente suficiente, lo que permite la colocación correcta del implante después de 6 meses de cicatrización (Maiorana et al.).

Lindhe et al. y Cecchinato reportaron la formación de hueso nuevo en un $26,9 \%$ mientras que el $25,6 \%$ representaba partículas residuales en la realización de preservaciones alveolares utilizando xenoinjerto bovino desproteinizado a los 6 meses de cicatrización (Lindhe et al., 2014).

El objetivo del presente estudio fue comparar los cambios dimensionales mediante el análisis en tomografía computarizada de haz cónico en sitios pre y post preservados con técnica Bartee y Bio-Col utilizando xenoinjerto InterOss a los 6 meses de cicatrización.

\section{MATERIAL Y MÉTODO}

Paciente masculino de 62 años, el cual es referido a la Clínica de Posgrado de Periodoncia UPAEP para extracciones múltiples. Posterior a la anamnesis no se encontró ningún dato relevante relacionado a su estado de salud sistémico por lo cual fue considerado paciente ASA I. Se continuó con la fase I del tratamiento periodontal y se solicitaron exámenes de laboratorio preoperatorios (química sanguínea de 6 elementos, biometría hemática, tiempo de protrombina y tiempo parcial de tromboplastina). Se registraron mediciones obtenidas mediante la tomografía computarizada de haz cónico inicial previa a la realización de preservaciones alveolares y se tomó la segunda tomografía a los 6 meses de cicatrización para poder observar la densidad ósea obtenida, y la medición en sentido vertical y horizontal, coincidiendo en el plano de corte. Al paciente se le realizaron un total 6 preservaciones alveolares en dientes uniradiculares, indicados para extracción con diagnóstico periodontal sin esperanza, los cuales no presentaron un proceso infeccioso activo para la posterior colocación y rehabilitación con implante dental; los sitios preservados se dividieron en dos grupos; en el Grupo A se usó la técnica de preservación alveolar Bartee $(n=3)$ y en el Grupo B se usó la técnica de preservación alveolar Bio-Col $(n=3)$. Se establecieron los siguientes criterios de selección para cada técnica; Grupo A se incluyó aquellos órganos dentarios con buena cantidad de tejido óseo remanente (mínimo $4 \mathrm{~mm}$ en sentido apicocoronal) que presentaran defecto en la pared vestibular. Grupo B se incluyeron aquellos órganos dentarios que presentaron alveolo íntegro (4 paredes remanentes) y una buena calidad de tejido óseo remanente (mínimo $4 \mathrm{~mm}$ en sentido apico-coronal).

El paciente firmó previo consentimiento informado, se procedió a realizar asepsia y antisepsia con yodo povidona, se anestesiaron (articaina $4 \%$ / epinefrina 1:100 000) los órganos dentarios Grupo $A$ $(11,21$ y 22$)$ y Grupo B $(13,23$ y 32). Se realizó la extracción atraumática, se lavó y se curetearon los alveolos; y se prosiguió con la técnica de preservación alveolar indicada en cada caso (Fig. 1).

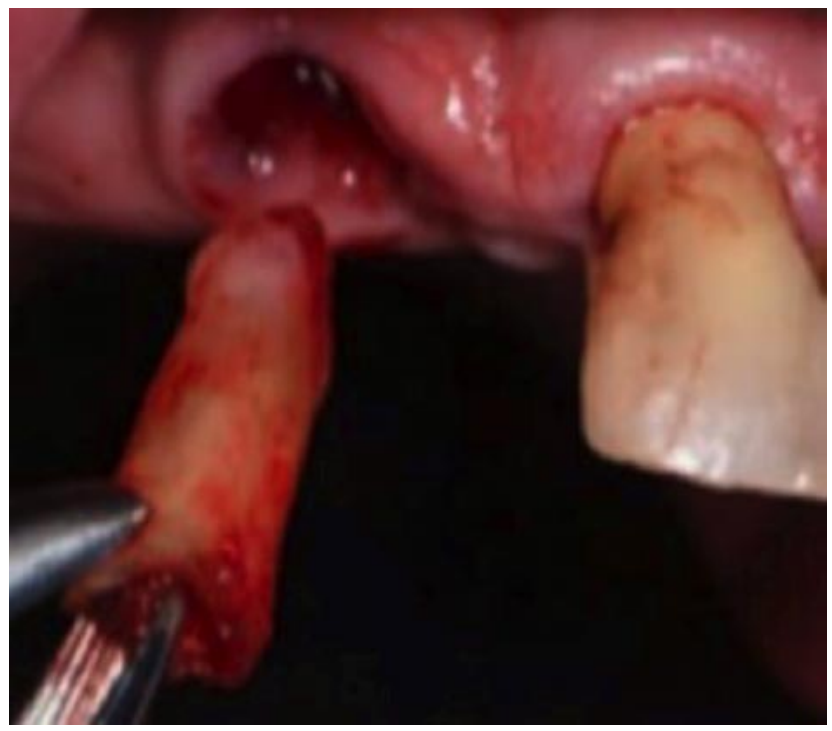

Fig. 1. Extracción atraumática.

En el caso del Grupo A se hidrató el xenoinjerto (InterOss ${ }^{\circledR}$ Anorganic Cancellous Bone Graft Granules 0,25-1 $\mathrm{mm}$ Sigma graft) con solución fisiológica en un godete estéril. El injerto óseo se llevó al sitio de extracción y se compactó suavemente en la totalidad del alveolo. Se evitó realizar una compactación excesiva del injerto ya que esto solo obstaculiza la revascularización del sitio (Fig. 2). 


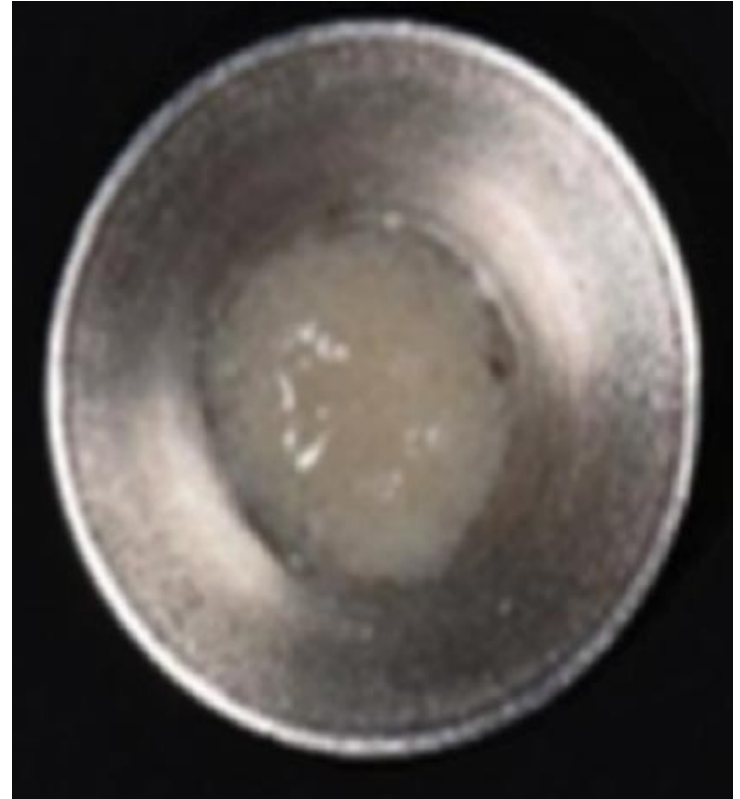

Fig. 2. Hidratación de xenoinjerto.

Se utilizó una membrana no reabsorbible de politetrafluoroetileno denso (Cytoplast ${ }^{\mathrm{TM}}$ Regentex TXT-200 singles, Osteogenics Biomedical Inc, Lubbock, Texas). Se estabilizó con puntos interrumpidos en el espacio interdental, papilas y se colocaron puntos de sutura colchonero horizontal a través de la abertura del alveolo. El material de sutura usado fue ácido poliglicólico (vicryl 4.0 ® poliglactina 910 ).

A los 21 días se realizó el retiro de la membrana (PTFE-d) con ayuda de unas pinzas de curación sin necesidad de infiltrar anestésico local (Fig. 3).
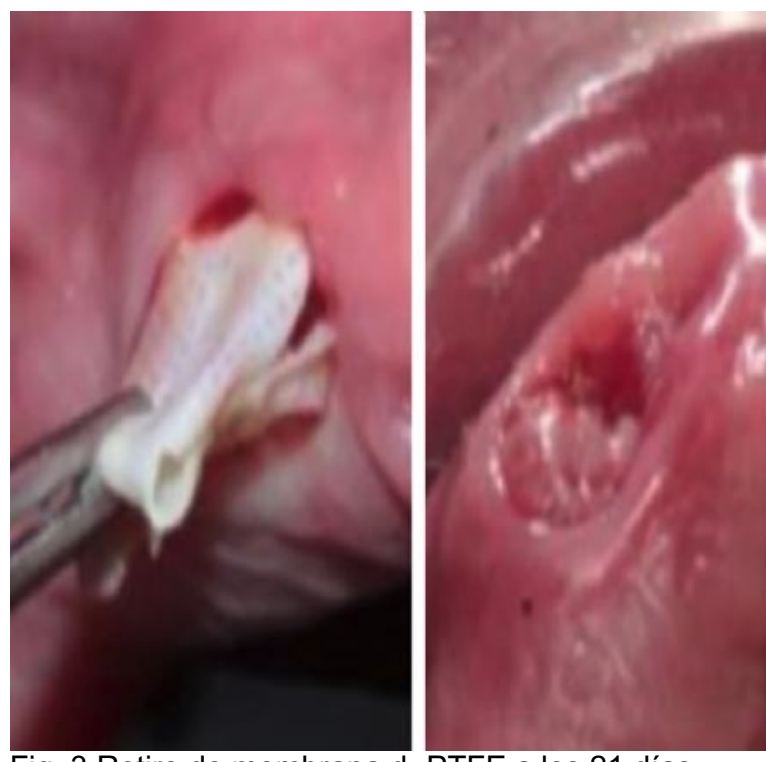

Fig. 3 Retiro de membrana d- PTFE a los 21 días.
En el Grupo B se colocó el injerto óseo en 3/4 del alveolo y el último $1 / 4$ fue ocupado por apósito de colágeno reabsorbible (CollaPlug ${ }^{\circledR}$ Zimmer biomet). Se colocaron puntos de sutura en cruz con sutura ácido poliglicólico (vicryl $4.0 \AA$ poliglactina 910 ), se estabilizó la sutura con adhesivo tisular (Periacryl 90HV® GluStitch Inc).

Concluido el procedimiento se le proporcionó al paciente por escrito las indicaciones y cuidados postoperatorios, así como la terapia antibiótica (amoxicilina $500 \mathrm{mg} 1$ cada 8 horas por 7 días) y el uso de colutorio con clorhexidina al 0,12 \% para evitar la colonización bacteriana de la zona tratada durante 15 días.

Previa a la realización de las extracciones y preservaciones se registraron mediciones obtenidas mediante la tomografía computarizada de haz cónico y a los 6 meses de cicatrización, se realizaron los mismos cortes coincidiendo en el mismo plano con una segunda tomografía (Fig. 4 y 5).

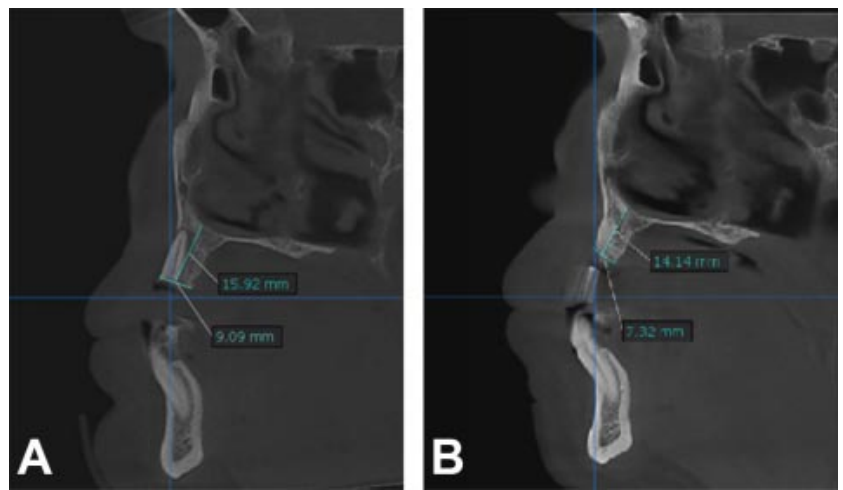

Fig. 4. Tomografía Computarizada de Haz Cónico (CBCT) grupo A (técnica preservación alveolar Bartee). A. medida basal. B. 6 meses cicatrización

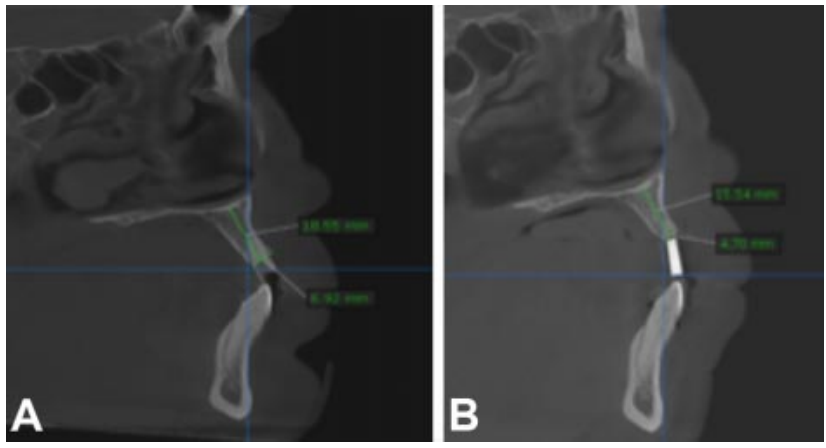

Fig. 5. Tomografía Computarizada de Haz Cónico (CBCT) grupo B (técnica preservación alveolar Bio-Col). A. medida basal. B. 6 meses cicatrización

Los datos se anexaron en una base de datos en el paquete estadístico IBM SPSS Statistics para Windows, versión 25 (IBM Corp., Armonk, N.Y., USA). 
Se aplicó la prueba Wilcoxon para muestras relacionadas para la comparación de medianas entre la medición basal y la medición a los 6 meses dentro de cada técnica, así como también se utilizó la prueba $U$ de Mann- Whitney para la comparación de medianas entre ambas técnicas. La significancia estadística se estableció en 0,05.

\section{RESULTADOS}

Al realizar el análisis dimensional mediante CBCT en sitios pre y post preservados con técnica de Bartee y Bio-Col utilizando xenoinjerto InterOss $₫$ a los 6 meses de cicatrización, se observó en el Grupo A menor reabsorción en sentido vertical en comparación al Grupo B de acuerdo con los resultados obtenidos representados en porcentajes de reabsorción (Fig. 6). Se realizó la comparación de la altura y anchura basal a los 6 meses por técnica (Tablas I y II) así como la comparación de la altura y anchura entre ambas técnicas (Tablas III y IV) con un intervalo en el porcentaje de reabsorción en altura de $11,24 \%-16,18 \%$ y anchura de $11,23-18,3 \%$ en los cuales no se observa una diferencia en la distribución de los datos, demostrando así, que no existe diferencia estadísticamente significativa entre ambas técnicas $(p>0,05)$.

Fig. 6. Porcentaje de reabsorción a los 6 meses de cicatrización.

Tabla I. Comparación de la altura basal y a los 6 meses por técnicas.

\begin{tabular}{llllllllll}
\hline & \multicolumn{3}{c}{ Altura Basal $(\mathrm{mm})$} & \multicolumn{4}{c}{ Altura a los 6 meses $(\mathrm{mm})$} & \multicolumn{2}{c}{ Valor $p$} \\
& Me dia & D.E. & Mediana & Rango & Me dia & D.E. & Me diana & Rango \\
\hline Bartee & 20,823 & 7,286 & 19,570 & 14,04 & 19,176 & 8,405 & 14,510 & 14,14 & 0,109 \\
Bio-Col & 17,160 & 0,135 & 16,720 & 2,14 & 14,806 & 0,481 & 14,580 & 0,88 & \\
\hline
\end{tabular}

*Prueba de Wilcoxon

Tabla II. Comparación de la anchura basal y a los 6 meses por técnicas.

\begin{tabular}{lllllllll}
\hline & \multicolumn{2}{l}{ Anchura Basal $(\mathrm{mm})$} & & \multicolumn{4}{c}{ Anchura a los 6 meses (mm) } \\
& Media & D.E. & Mediana & Rango & Media & D.E. & Mediana & Rango \\
\hline Bartee & 8,626 & 0,645 & 8,900 & 0,20 & 6,846 & 0,382 & 7,620 & 2,42 \\
Bio-Col & 7,66 & 0,913 & 7,760 & 0,82 & 6,496 & 0,242 & 6,340 & 2,47 \\
\hline
\end{tabular}

Tabla III. Comparación de la altura entre técnicas.

\begin{tabular}{|c|c|c|c|c|c|}
\hline & \multicolumn{2}{|c|}{ Bartee } & \multicolumn{2}{|c|}{ Bio-Col } & \multirow[t]{2}{*}{ Valor $p$} \\
\hline & Me diana & Rango & Me diana & Rango & \\
\hline Altura basal (mm) & 19,570 & 14,04 & 16,720 & 0,513 & 0,513 \\
\hline Altura 6 meses $(\mathrm{mm})$ & 14,510 & 14,14 & 14,580 & 0,827 & 0,827 \\
\hline \% de reabsorción & 10,240 & 22,22 & 16,180 & 0,275 & 0,275 \\
\hline
\end{tabular}

Tabla IV. Comparación de la anchura entre técnicas.

\begin{tabular}{llllll}
\hline & Mediana & Bartee & \multicolumn{2}{c}{ Bio-Col } & Valor $p$ \\
& Rango & Mediana & Rango & \\
\hline Anchura basal $(\mathrm{mm})$ & 8,900 & 0,20 & 7,760 & 0,82 & 0,127 \\
Anchura 6 meses $(\mathrm{mm})$ & 7,620 & 2,42 & 6,340 & 2,47 & 0,827 \\
\% de reabsorción & 10,230 & 12,84 & 18,300 & 10,97 & 0,827 \\
\hline${ }^{*} \mathrm{U}$ de Mann-Whitney & \multicolumn{1}{c}{} \\
\hline
\end{tabular}




\section{DISCUSIÓN}

En un ensayo clínico controlado aleatorizado se realizó evaluación dimensional en diferentes técnicas de preservación alveolar en sector posterior $(n=35)$ en 4 grupos de estudio utilizando DBBM. Grupo T1 se colocó DBBM en conjunto injerto de tejido blando obtenido del paladar, T2 únicamente se colocó DBBM, T3 se colocó DBBM asistido por una membrana de colágeno reabsorbible, T4 no se realizó ningún tratamiento. A la valoración posterior a 6 meses de cicatrización se observó que no existía diferencia estadísticamente significativa $(p>0,05)$ en cambios dimensionales $(<1 \mathrm{~mm})$, el contorno vestibular presentaba menores cambios dimensionales en el Grupo T1, a diferencia del Grupo T4 que presentó cambios (2 $\mathrm{mm}$ ). En la presente investigación, se observó que no existe diferencia estadísticamente significativa $(p>0,05)$, en cuanto a cambios dimensionales en técnica de preservación alveolar Bartee y Bio-col a los 6 meses de cicatrización utilizando xenoinjerto, coincidiendo así con lo reportado por Fischer et al. (2018).

Tan et al. Reportaron el porcentaje de cambios dimensionales en una revisión sistemática posterior a la extracción dental sin intervenir en el proceso de cicatrización en sentido vertical oscila entre un $11 \%$ al $22 \%$ y en sentido horizontal del $29 \%$ al $63 \%$ tan sólo a los 6-7 meses de cicatrización, en cambio al utilizar xenoinjerto en preservación alveolar utilizando técnica Bartee o Bio-col se pudo observar la estabilidad dimensional del alveolo presentando un intervalo en sentido vertical entre un $13,58 \%-20,76 \%$ y en sentido horizontal del $13,45 \%-15,72 \%$ a los 6 meses de cicatrización, obtenidos en el presente estudio.

Por otra parte, en una revisión sistemática de 32 estudios, 1354 preservaciones alveolares referentes al volumen obtenido en el alveolo cicatrizado utilizando biomateriales como xenoinjerto, aloinjerto y aloplástico se reportó que el xenoinjerto y aloinjerto óseo mostraron menor pérdida dimensional que los alvéolos no injertados o injertados con aloplásticos, así como también el uso de xenoinjerto para la realización de preservación alveolar era el más reportado y estudiado ( 21 de los 32 estudios analizados) con resultados predecibles a corto plazo, de igual manera su combinación con una membrana como barrera o colágeno reabsorbible para el correcto sellado y aislado del alvéolo, por lo que se sugiere el uso de xenoinjerto para la realización de preservación alveolar (Jambhekar et al., 2014). Asímismo, Serrano Méndez et al. (2017) compararon aloinjerto versus xenoinjerto utilizado en preservación alveolar asistido por una membrana de colágeno reabsorbible, reportando que no existió diferencia estadísticamente significativa a los 6 meses de cicatrización entre ambos grupos. Por lo que se puede concluir en el presente estudio que la técnica de preservación alveolar Bartee asistida por una membrana texturizada PTFE-d y Bio-Col utilizando un apósito de colágeno reabsorbible en combinación con un xenoinjerto parece ser una opción predecible, proporcionando resultados alentadores a los 6 meses de cicatrización obteniendo la estabilidad de los tejidos circundante, los cuales permiten reducir más no evitar el proceso de reabsorción fisiológico.

\section{CONCLUSIÓN}

La preservación alveolar con xenoinjerto, proporciona una estabilidad dimensional debido a que contrarresta el proceso de reabsorción fisiológica, más no lo evita, asegurando la estabilidad del tejido circundante siendo una opción predecible.

ELIZALDE, M. M. K.; HERNÁNDEZ, R. C.; ROCHA, R. V. M.; MAYORAL, G. V. A. Dimensional changes in bartee and bio-col ridge preservation technique using xenograft interOss ${ }^{\circledR}$. Int. J. Odontostomat., 15(2):370-376,2021.

ABSTRACT: Preserving the dimensions and contour of the alveolar ridge after the dental extraction, it's of utmost importance to avoidsubsequent problems for the placement of a dental implant. The objective of this study was compare the dimensional changes through the analysis in Cone-beam computed tomography (CBCT), in pre and post sites preserved with the Bartee and Bio-Col technique with xenograft at 6 months of healing. In a 62-year-old patient, 6 alveolar preservations were performed in uniradicular dental organs, with a hopeless periodontal diagnosis, group A the Bartee alveolar ridge preservation technique $(n=3)$ and group $B$ the Bio-Col alveolar ridge preservation technique ( $\mathrm{n}$ = 3). Xenograft (InterOss $®$ Anorganic Cancellous Bone Graft Granules $0.25-1 \mathrm{~mm}$ Sigma graft) was used in both groups. In the group A a bone graft was placed in the entire socket, assisted by a dense non-absorbable polytetrafluoroethylene membrane (Cytoplast TM Regentex TXT-200 singles, Osteogenics Biomedical Inc, Lubbock, Texas). In the group $B$ the bone graft was placed in 3/4 of the socket and the last $1 / 4$ of the socket was occupied by an absorbable collagen dressing (CollaPlug $₫$ Zimmer biomet). Measurements obtained by initial CBCT before recording alveolar ridge preservations were recorded, and the second CBCT was taken 6 months after healing obtaining the measurement 
vertically and horizontally, coinciding in the section plane. It was observed through dimensional analysis in group $A$ and Group B, obtaining $13.58 \%$ and $20.76 \%$ of reabsorption vertically and $13.45 \%$ and $15.72 \%$ respectively at 6 months of healing, using xenograft, so there is no statistical difference significant in terms of dimensional changes between both techniques $p>0.05$. Alveolar ridge preservation provides dimensional stability by counteracting the physiological resorption process, being a predictable option.

KEY WORDS: CBCT, alveolar bone loss, bone remodeling, heterograft.

\section{REFERENCIAS BIBLIOGRÁFICAS}

Bartee, B. K. Extraction site reconstruction for alveolar ridge preservation. Part 1: rationale and materials selection. J. Oral Implantol., 27(4):187-93, 2001,

Bartee, B. K. Extraction site reconstruction for alveolar ridge preservation. Part 2: membrane-assisted surgical technique. J. Oral Implantol., 27(4):194-7, 2001.

Chan, H. L.; Lin, G. H.; Fu, J. H. \& Wang, H. L. Alterations in bone quality after socket preservation with grafting materials: a systematic review. Int. J. Oral Maxillofac. Implants, 28(3):71020,2013

Fischer, K. R.; Mühlemann, S.; Jung, R. E.; Friedmann, A. \& Fickl, $S$. Dimensional evaluation of different ridge preservation techniques with a bovine xenograft: a randomized controlled clinical trial. Int. J. Periodontics Restorative Dent., 38(4):549-56, 2018.

Fotek, P. D.; Neiva, R. F. \& Wang, H. L. Comparison of dermal matrix and polytetrafluoroethylene membrane for socket bone augmentation: a clinical and histologic study. J. Periodontol., 80(5):776-85, 2009.

Fowler, E. B. \& Whicker, R. Modified approach to the Bio-Col ridge preservation technique: a case report. J. Contemp. Dent. Pract., 5(3):82-96, 2004.

Hämmerle, C. H; Araújo, M. G.; Simion, M. \& Osteology Consensus Group 2011. Evidence-based knowledge on the biology and treatment of extraction sockets. Clin. Oral Implants Res., 23 Suppl. 5:80-2, 2012.

Jamjoom, A. \& Cohen, R. E. Grafts for ridge preservation. J. Funct. Biomater., 6(3):833-48, 2015.

Lindhe, J.; Cecchinato, D.; Donati, M.; Tomasi, C. \& Liljenberg, B. Ridge preservation with the use of deproteinized bovine bone mineral. Clin. Oral Implants Res., 25(7):786-90, 2014.

Maiorana, C.; Poli, P. P.; Deflorian, M.; Testori, T.; Mandelli, F.; Nagursky, H. \& Vinci, R. Alveolar socket preservation with demineralised bovine bone mineral and a collagen matrix. J. Periodontal Implant Sci., 47(4):194-210, 2017.

Position Paper: Periodontal Regeneration. J. Periodontol., 76(9):1601-22, 2005.

Sclar, A. G. Preserving alveolar ridge anatomy following tooth removal in conjunction with immediate implant placement. The Bio-Col technique. Atlas Oral Maxillofac. Surg. Clin. North Am., 7(2):3959, 1999.

Serrano Méndez, C. A.; Lang, N. P.; Caneva, M., Ramírez Lemus, G.; Mora Solano, G. \& Botticelli, D. Comparison of allografts and xenografts used for alveolar ridge preservation. A clinical and histomorphometric RCT in humans. Clin. Implant Dent. Relat. Res., 19(4):608-15, 2017.
Shakibaie-M, B. Comparison of the effectiveness of two different bone substitute materials for socket preservation after tooth extraction: a controlled clinical study. Int. J. Periodontics Restor. Dent., 33(2):223-8, 2013.

Tan, W. L.; Wong, T. L. T.; Wong, M. C. M. \& Lang, N. P. A systematic review of post-extractional alveolar hard and soft tissue dimensional changes in humans. Clin. Oral. Implants Res., 23 Suppl. 5:1-21, 2012.

Wang, H. L.; Kiyonobu, K. \& Neiva, R. F. Socket augmentation: rationale and technique. Implant Dent., 13(4):286-96, 2004.

Dirección para correspondencia:

Melanie Karoly Elizalde Mota

Residente Posgrado Periodoncia

Universidad Popular Autónoma de Puebla

Calle 23 Sur \#1102

Barrio de Santiago, 72410

Puebla, Pue.

MÉXICO

Email: melaniek_elizalde@live.com.mx 